Pedro Gabino Mendoza Pacheco

\title{
Análise Numérica do Fluxo e da Estabilidade de uma Pilha de Lixiviação de Minério de Cobre
}

Dissertação de Mestrado

Dissertação apresentada como requisito parcial para obtenção do título de Mestre pelo Programa de PósGraduação em Engenharia Civil da PUC-Rio. Área de concentração: Geotecnia

Orientador: Celso Romanel Co-orientador: Denys Parra Murrugarra (UNI-Peru) 


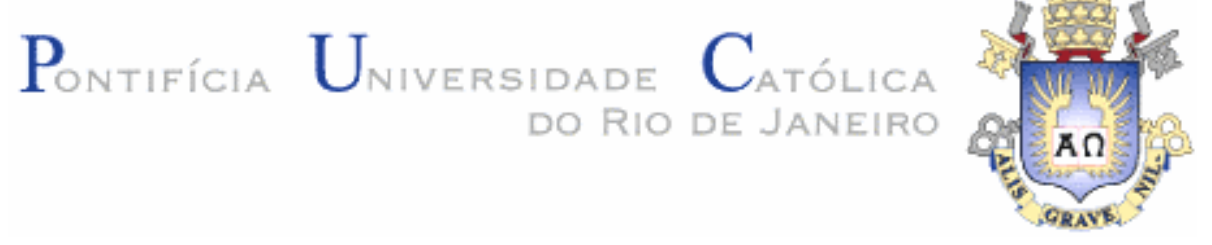

Pedro Gabino Mendoza Pacheco

\section{Análise Numérica do Fluxo e da Estabilidade de uma Pilha de Lixiviação de Minério de Cobre}

Dissertação apresentada como requisito parcial para obtenção do título de Mestre pelo Programa de PósGraduação em Engenharia Civil da PUC-Rio. Aprovada pela Comissão Examinadora abaixo assinada.

Celso Romanel Orientador, PUC - Rio

Deane de Mesquita Roehl PUC - Rio

\section{Anna Paula Lougon Duarte} PUC - Rio

José Eugênio Leal Coordenador Setorial do Centro Técnico Científico da PUC-Rio

Rio de Janeiro, Agosto de 2005 
Todos os direitos reservados. É proibida a reprodução total ou parcial do trabalho sem autorização da universidade, do autor e do orientador.

\section{Pedro Gabino Mendoza Pacheco}

Graduou-se em Engenharia Civil pela Universidade Nacional de Engenharia (UNI-Peru) em 1996. Desenvolveu durante a tese de graduação o programa computacional denominado GeoDam para estudo do comportamento de barragens. Atuou na seção geotécnica do Centro de Investigações Sísmicas e Mitigação de Desastres (CISMIDUNI) em Lima, Peru. Ingressou em 2003.2 no curso de mestrado em Engenharia Civil da Pontifícia Universidade Católica do Rio de Janeiro, na área de Geotecnia, desenvolvendo dissertação de mestrado na linha de pesquisa Geomecânica Computacional para análise numérica do fluxo e da estabilidade de uma pilha de lixiviação de minério de cobre.

\section{Ficha Catalográfica}

\section{Mendoza, Pedro Pacheco.}

Análise numérica do fluxo e da estabilidade de uma pilha de lixiviação de minério de cobre / Pedro Mendoza Pacheco; orientador: Celso Romanel; co-orientador: Denys Parra Murrugarra - Rio de Janeiro: PUC, Departamento de Engenharia Civil, 2005.

$$
\text { v., } 129 \text { f.: il. ; } 29,7 \mathrm{~cm}
$$

Dissertação (mestrado) - Pontifícia Universidade Católica do Rio de Janeiro, Departamento de Engenharia Civil.

Inclui referências bibliográficas.

1. Engenharia civil - Tese. 2. Pilha de lixiviação. 3. Fluxo não-saturado. 4. Estabilidade sísmica de taludes. 5. Elementos finitos. I. Romanel, Celso. II. Murrugarra, Denys Parra. III. Pontifícia Universidade Católica do Rio de Janeiro. Departamento de Engenharia Civil. IV Título. 
Para meus pais, Matilde e Augusto, pelo apoio de sempre; para mia esposa Ingrid e meu filho Pedro Manuel por seu grande amor e compreensão; aos meus irmãos pelo grande amor com que sempre me brindam. 


\section{Agradecimentos}

A Deus, por todas as graças recebidas.

À Pontifícia Universidade Católica (PUC-Rio) e ao CNPq por terem me concedido a oportunidade de realizar este trabalho.

Ao professor Celso Romanel, por sua orientação, confiança e apoio, meus sinceros agradecimentos.

A meu co-orientador Denys Parra, pela sincera amizade.

A meus pais Matilde e Augusto, pelo apoio de sempre.

Aos meus irmãos Luz, Javier, Eufemia, Gilmer, Orlando, Wilfredo, Consuelo, Yolanda e Luis pelo grande amor com que sempre me brindam.

A mia esposa Ingrid e meu filho Pedro Manuel por seu grande amor e compreensão.

A meus amigos e companheiros de estudo do curso de Mestrado em Engenharia Civil da PUC-Rio.

Aos funcionários da Secretaria do Departamento de Engenharia Civil, especialmente à Ana Roxo, pela dedicação e paciência com os alunos de pósgraduação, principalmente com os estrangeiros. 


\section{Resumo}

Mendoza, Pedro Pacheco; Romanel, Celso; Murrugarra, Denys Parra. Análise numérica do fluxo e da estabilidade de uma pilha de lixiviação de minério de cobre. Rio de Janeiro, 2005. 129p. Dissertação de Mestrado - Departamento de Engenharia Civil, Pontifícia Universidade Católica do Rio de Janeiro.

Esta dissertação apresenta uma análise numérica do fluxo não saturado em uma pilha de lixiviação de minério de cobre. Adicionalmente, foi feito também um estudo da estabilidade estática e sísmica da pilha de lixiviação, por sua construção estar planejada em região de alta sismicidade no sul do Peru. A pilha será construída com minério de cobre não tratado sobre base impermeável para evitar a contaminação do meio ambiente por fluxo da solução ácida através da fundação. Os resultados obtidos nas análises numéricas indicam que a pilha de lixiviação apresenta fatores de segurança satisfatórios considerando sismos de magnitude até 7,5 com aceleração máxima de até 0,30g. Da mesma forma, constatou-se que a posição da linha freática formada pela solução de lixiviação não atinge as camadas previstas para proteção das tubulações de drenagem.

\section{Palavras - chave}

Pilha de lixiviação, fluxo não-saturado, estabilidade sísmica de taludes, elementos finitos. 


\section{Abstract}

Mendoza, Pedro Mendoza; Romanel, Celso (advisor); Murrugarra, Denys Parra (co-advisor). Numerical analysis of the flow and stability of a copper ore heap leach. Rio de Janeiro, 2005. 129p., M.Sc. Thesis - Department of Civil Engineering, Pontifícia Universidade Católica do Rio de Janeiro.

This dissertation presents a numerical analysis for the non saturated flow throughout a copper ore heap leach. Additionally, static and dynamic stability analyses were also made because the heap leach is planned to be built in a highly seismic region in the south of Peru. The heap will be constructed with non-treated copper ore (run of mine) on impermeable pad specially devised to avoid any possibility of ground contamination by the flow through the foundation of the acid solution used for the lixiviation process. The numerical results obtained in the stability analyses indicate that the heap leach presents satisfactory safety factors even when considering earthquakes of magnitude 7.5 with maximum acceleration of $0.30 \mathrm{~g}$. It has been also observed that the phreatic line formed by the lixiviation fluid does not reach the layers devised to protect the drainage pipes of the lixiviation system.

\section{Keywords}

Heap leach, unsaturated fluid flow, slope seismic stability; finite elements. 


\section{Sumário}

1 Introdução $\quad 21$

2 Pilhas de lixiviação $\quad 24$

2.1. Introdução 24

2.2. Componentes de uma pilha de lixiviação 24

2.2.1. Fonte de minério $\quad 24$

2.2.2. Preparação do minério 25

2.2.3. Pilha e base (pad) 26

2.2.4. Aplicação da solução / sistema de coleta 28

2.2.5. Piscinas de solução fértil e estéril 29

2.3. Aspectos Geotécnicos 29

2.3.1. Base da pilha (pad) 30

2.3.2. Piscinas de solução fértil e estéril (ponds) 32

2.3.3. Sistema de coleta 32

2.3.4. Pilha 34

2.4. Estabilidade de taludes $\quad 34$

2.5. Resistência ao cisalhamento 37

3 Relações constitutivas para fluxo em meios não saturados 40

3.1. Introdução 40

3.2. Determinação direta da função de condutividade hidráulica 41

3.3. Determinação indireta da função de condutividade hidráulica 42

3.3.1. Método de Fredlund, Xing e Huang (1994) 43

3.3.2. Método de Green e Corey ( 1971) 45

3.3.3. Método de van Genuchten (1980) 46

3.4. Determinação indireta da função de teor de umidade volumétrico 47

3.4.1. Método de Arya e Paris (1981) 48

3.4.2. Método Modificado de Kovacs (2001) 49

3.4.3. Método de Fredlund e Xing (1994) 52 
4 Estabilidade de taludes 54

4.1. Métodos de equilíbrio limite 54

4.1.1. Método das fatias 55

4.1.2. Solos não saturados $\quad 59$

4.2. Método dos elementos finitos 60

4.2.1. Método direto: simulação do colapso 62

4.2.2. Método indireto: equilíbrio limite aperfeiçoado 63

4.3. Análise sísmica 66

4.3.1. Análise pseudo-estática 66

4.3.2. Método de Newmark (1965) 66

5 Análise do comportamento de pilha de lixiviação 69

5.1. Modelagem da pilha de lixiviação 69

5.2. Propriedades dos materiais $\quad 71$

5.2.1. Resultados de ensaios no minério de cobre 71

5.2.2. Resultados de ensaios no material das camadas de proteção 74

5.3. Avaliação aproximada do espaçamento entre drenos 77

5.4. Análise numérica do fluxo não saturado 2D 79

5.5. Análises de estabilidade 88

5.5.1. Análise pelo método dos elementos finitos 90

5.5.2. Análise pelo método de equilíbrio limite aperfeiçoado 96

5.5.3. Análise pelo método de equilíbrio limite 97

5.5.4. Comparação dos resultados 98

5.5.5. Análise pseudo-estática 98

5.6. Análise sísmica 99

5.6.1. Terremoto de Lima (1974) 106

5.6.2. Terremoto de Moquegua (2001) 110

5.7. Estabilidade estática pós sismo 112

5.7.1. Análise pelo método dos elementos finitos 112

5.7.2. Análise pelo método de equilíbrio limite 118

6 Conclusões e sugestões 120 
6.1. Conclusões 120

6.2. Sugestões 122

7 Referências bibliográficas 123

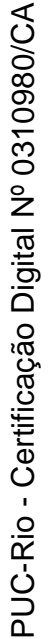




\section{Lista de figuras}

Figura 2.1 - Esquema do processo de uma pilha de lixiviação (Dorey, Zyl e Kiel, 1988).

Figura 2.2 - Pilha com base re-utilizável (Dorey, Zyl e Kiel, 1988). 27

Figura 2.3 - Pilha com base expansível (Dorey, Zyl e Kiel, 1988). 27

Figura 2.4 - Pilha de lixiviação em vale (Dorey, Zyl e Kiel, 1988). 28

Figura 2.5 - Características gerais das instalações de uma pilha de lixiviação (Strachan e Dorey, 1988) 30

Figura 2.6 - Aspectos gerais do local de construção da pilha ((Strachan e Dorey, 1988)

Figura 2.7 - Aspectos gerais do sistema de coleta (Strachan e Dorey, 1988) 33

Figura 2.8 - Potenciais superfícies de ruptura na pilha ( Harper; Leach, Tape 1987)

Figura 2.9 - Ângulo de atrito versus tensão vertical (psi) - (Leps, 1970). 38

Figura 2.10 - influência da técnica de construção na estabilidade da pilha: a) empilhamento para jusante; b) empilhamento para montante (Smith e Giroud, 2000).

Figura 3.1 - Função de condutividade hidráulica (Fredlund e Rahardio, 1993). 41

Figura 3.2 - Função do teor de umidade volumétrico (Fredlund e Rahardio, 1993).

Figura 3.3 - Funções do teor de umidade volumétrico para areia fina, silte e argila (Ho, 1979)

Figura 3.4 - Curva de adsorção e dessorção para um solo de silte (Fredlund, Xing e Huang, 1994)

Figura 4.1 - Forças atuantes em uma fatia vertical de uma superfície de deslizamento (GEO - SLOPE/W) 56

Figura 4.2: Tensões atuantes na superfície potencial de ruptura 65

Figura 4.3: Distribuição de tensões cisalhantes ( $\tau$ e s) ao longo da superfície potencial de ruptura $(\mathrm{A} \rightarrow \mathrm{B}) \quad 65$

Figura 4.4 - Analogia de Newmark (1965) entre uma massa de solo 
potencialmente instável e o bloco rígido sobre um plano inclinado.

Figura 4.5 - Integrações no tempo para determinação da velocidade e deslocamento relativos pelo método de Newmark (Smith, 1995).

68

Figura 5.1- Esquema da modelagem da pilha de lixiviação de minério de cobre. 70 Figura 5.2.- Curvas características de sucção do minério de cobre da pilha de lixiviação. 72

Figura 5.3.- Função de condutividade hidráulica para a camada de minério 172 Figura 5.4.- Função de condutividade hidráulica para a camada de minério 273 Figura 5.5.- Função de condutividade hidráulica para a camada de minério 373 Figura 5.6.- Função de condutividade hidráulica para a camada de minério 473 Figura 5.7.- Função de condutividade hidráulica para a camada de minério 574 Figura 5.8.- Função de condutividade hidráulica para a camada de minério $6 \quad 74$ Figura 5.9.- Curvas características de sucção para a camada de proteção 275 Figura 5.10.- Curvas características de sucção para a camada de proteção 176 Figura 5.11.- Função de condutividade hidráulica para a camada de proteção 276 Figura 5.12.- Função de condutividade hidráulica para a camada de proteção 177 Figura 5.13.- Superfície freática devido à infiltração pela superfície 78 Figura 5.14- Ilustração de parte da malha de elementos finitos correspondente às duas camadas de proteção e camadas de minério 1 e 6.

80

Figura 5.15.- Ilustração dos vetores de velocidades de fluxo e posição da zona de saturação.

Figura 5.16.- Distribuição do teores de umidade volumétricos ao longo da altura da pilha.

Figura 5.17- Perfil de saturação nas camadas de proteção com a variação da vazão de aplicação da solução ácida. 82

Figura 5.18.- Linha freática e velocidades de fluxo para vazão $\mathrm{q}=8 \mathrm{l} / \mathrm{h} / \mathrm{m}^{2} \quad 83$

Figura 5.19.- Linha freática e velocidades de fluxo para vazão $\mathrm{q}=60 \mathrm{l} / \mathrm{h} / \mathrm{m}^{2} \quad 83$ Figura 5.20 - Perfil de saturação nas camadas de proteção com a variação do coeficiente de permeabilidade saturado da camada de proteção 2 .

Figura 5.21 - Posição da linha freática e vetores de velocidade de fluxo considerando coeficiente de permeabilidade saturado na camada 2 igual a $\mathrm{k}=$ $2.53 \times 10^{-3} \mathrm{~cm} / \mathrm{s}$.

Figura 5.22 - Posição da linha freática e vetores de velocidade de fluxo 
considerando coeficiente de permeabilidade saturado na camada 2 igual a $\mathrm{k}=$ $2.53 \times 10^{-4} . \mathrm{cm} / \mathrm{s}$

Figura 5.23- Perfil de saturação nas camadas de proteção com a variação do coeficiente de permeabilidade saturado da camada de proteção 1 .

Figura 5.24 - Posição da linha freática e vetores de velocidade de fluxo considerando coeficiente de permeabilidade saturado na camada 1 igual a $\mathrm{k}=$ $2.53 \times 10^{-3} \mathrm{~cm} / \mathrm{s}$ 86

Figura 5.25 - Posição da linha freática e vetores de velocidade de fluxo considerando coeficiente de permeabilidade saturado na camada 1 igual a $\mathrm{k}=$ $2.53 \times 10^{-4} \mathrm{~cm} / \mathrm{s}$ 86

Figura 5.26.- Malha de elementos finitos da seção transversal da pilha de lixiviação.

Figura 5.27.- Distribuição dos vetores das velocidades de fluxo, preponderantemente verticais

Figura 5.28.- Distribuição das poropressões nas zonas não saturadas e saturadas 89 Figura 5.29.- Distribuição dos teores de umidade volumétrico na pilha de lixiviação 90

Figura 5.30.- Distribuição das cargas totais 90

Figura 5.31.- Distribuição dos valores das tensões principais máximas na pilha de lixiviação.

Figura 5.32.- Convergência da solução numérica para valores do fator de redução M.

Figura 5.33.- Campo de deslocamentos para $\mathrm{M}=1,10$ a $\mathrm{M}=1,40$ 94

Figura 5.34.- Campo de deslocamentos para $\mathrm{M}=1$,45.a $\mathrm{M}=1.65$ com indicação das superfícies plana e composta para $\mathrm{M}=1,60$.. 95

Figura 5.35 .- Superfície de ruptura composta $(\mathrm{FS}=1,634)$

Figura 5.36.- Superfície de ruptura plana (FS = 1,645) 96

Figura 5.37.- Potencial superfície de ruptura composta - método de equilíbrio limite.

Figura 5.38.- Posição da potencial superfície de ruptura plana - método de equilíbrio limite.

Figura 5.39.- Função de redução do módulo de cisalhamento G. 100

Figura 5.40.- Função da redução da razão de amortecimento $\xi$. 
Figura 5.41.- Sismicidade na região sul do Peru entre 1964 e 1996 (magnitudes M $>$ 5) - Instituto Geofísico do Peru

Figura 5.42.- Registro do terremoto de Lima (1974) normalizado para aceleração máxima de $0.30 \mathrm{~g}$.

Figura 5.43.- Registro do terremoto de Moquega (2001) com aceleração máxima de $0.30 \mathrm{~g}$.

102

Figura 5.44.- Registro dos deslocamentos horizontais no topo da pilha

Figura 5.45.- Registro das acelerações horizontais no topo da pilha.

Figura 5.46.- Registro de deslocamentos horizontais no topo da pilha. 105

Figura 5.47.- Registro das acelerações horizontais no topo da pilha. 105

Figura 5.48.- Variação do fator de segurança no tempo $\left(F_{\max }=3,90, F_{\min }=1\right) .107$

Figura 5.49.- Variação do fator de segurança com a aceleração média da massa deslizante.

107

Figura 5.50.- Variação da aceleração média da massa deslizante em função do tempo.

Figura 5.51 - Variação do fator de segurança no tempo $\left(\mathrm{F}_{\max }=3,50, \mathrm{~F}_{\min }=1,05\right)$

Figura 5.52 - Variação do fator de segurança com a aceleração média da massa deslizante. .

Figura 5.53.- Variação da aceleração média como uma função do tempo

109

Figura 5.54 - Variação do fator de segurança médio da massa deslizante no tempo $\left(F_{\max }=3,20, F_{\min }=0,90\right)$.

Figura 5.55.- Variação do fator de segurança com a aceleração média da massa deslizante.

Figura 5.56.- Variação do fator de segurança médio da massa deslizante no tempo

$$
\left(\mathrm{F}_{\max }=3,05, \mathrm{~F}_{\min }=0,95\right) .
$$

Figura 5.57.- Variação do fator de segurança com a aceleração média da massa deslizante.

Figura 5.58.- Convergência da solução numérica para variação do fator de redução $\mathrm{M}$

Figura 5.59.- Campos de deslocamentos para $\mathrm{M}=1 \mathrm{a} \mathrm{M}=1.25$, com indicação da superfície plana para $\mathrm{M}=1.20$

Figura 5.60 - Convergência da solução numérica para variação do fator de redução 
M.

Figura 5.61.- Campos de deslocamentos para o fator de redução $\mathrm{M}=1$ a $\mathrm{M}=$ 1,25, com indicação da superfície plana para $M=1.20$.

117

Figura 5.62.- Posição das superfícies de ruptura nas análise pré e pós - sismo 118 


\section{Lista de tabelas}

Tabela 4.1 - Características dos MEL não rigorosos (de Campos, 1985). 58

Tabela 4.2 - Características dos métodos de equilíbrio limite rigorosos (de Campos, 1985) 59

Tabela 4.3 - Valores típicos do coeficiente sísmico $\mathrm{k}_{\mathrm{h}} \quad 66$

$\begin{array}{ll}\text { Tabela 5.1.-Resumo das propriedades do minério } & 71\end{array}$

Tabela 5.2.-Resumo das propriedades do material das camadas de proteção 75

Tabela 5.3.- Altura de saturação para diferentes espaçamentos entre drenos, coeficientes de permeabilidade da camada 1 de proteção do revestimento e $\begin{array}{ll}\text { vazões de aplicação da solução. } & 78\end{array}$

Tabela 5.4.- Comparação da altura máxima de saturação (m) determinadas $\begin{array}{ll}\text { analiticamente e numericamente. } & 79\end{array}$

Tabela 5.5. Características das 22 modelagens numéricas. 80

Tabela 5.6.- Altura máxima da zona de saturação considerado fluxo 2D não saturado. $\quad 87$

Tabela 5.7.- Parâmetros do modelo de Van Genuchten (1980) 87

Tabela 5.8.- Valores dos parâmetros de resistência (condição saturada) e peso específico natural das camadas de minério de cobre. 88

Tabela 5.9.- Redução da resistência das camadas de minério na simulação do colapso. $\quad 90$

Tabela 5.10.- Redução da resistência das camadas de minério na simulação do colapso. 91

Tabela 5.11.- Valores dos parâmetros para as análises de tensões elastoplásticas.

Tabela 5.12.- Fatores de segurança para superfície composta 97

Tabela 5.13.- Fator de segurança para superfície plana. 98

Tabela 5.14.- Valores dos fatores de segurança estáticos 98

Tabela 5.15.- Fatores de segurança para superfície composta 99

Tabela 5.16.- Fatores de segurança para superfície plana 99

Tabela 5.17.- Parâmetros para análise dinâmica. 100 
Tabela 5.18 .-Comparação dos fatores de segurança dinâmicos avaliados com os registros dos terremotos de Lima (1974) e de Moquegua (2001) 112

Tabela 5.19 .-Redução do ângulo de resistência ao cisalhamento na simulação do colapso. 113

Tabela 5.20 .-Fator de segurança pós sismo para superfície composta 119

Tabela 5.21 .-Fator de segurança pós sismo para superfície plana 119

Tabela 5.22 .-Fator de segurança pós sismo para superfície composta. 119

Tabela 5.23 .-Fator de segurança pós sismo para superfície plana. 119 

Lista de Símbolos
$\theta$
$\theta_{\mathrm{s}}$
$\theta_{\mathrm{r}}$
$\psi$
$\mathrm{k}_{\mathrm{s}}$
$\kappa_{\omega}$
$\mathrm{m}_{\mathrm{v}}$
a
n
m
$\mathrm{C}(\psi)$
$\mathrm{C}_{\mathrm{r}}$
$\mathrm{k}(\theta)_{\mathrm{i}}$
$\mathrm{k}_{\mathrm{s}} / \mathrm{k}_{\mathrm{sc}}$
$\mathrm{k}_{\mathrm{sc}}$
$\mathrm{T}$
$\beta$
$\eta$
$\gamma_{\omega}$
g
$\mathrm{S}_{\mathrm{p}}$
$\mathrm{V}_{\mathrm{w}}$
V
$\mathrm{V}_{\mathrm{i}} \quad$ Volume de poros por unidade de massa de um segmento
$\mathrm{W}_{\mathrm{i}} \quad$ Massa sólida por unidade de massa de um segmento
Teor de umidade volumétrico
Teor de umidade volumétrico saturado
Teor de umidade volumétrico residual
Sucção mátrica
Coeficiente de permeabilidade saturado
Coeficiente de permeabilidade não saturado
Coeficiente de compressibilidade volumétrica
Valor aproximado de entrada de ar
Parâmetro controla pendeiente do ponto de inflexão da função $\theta$
Parâmetro que descreve o teor de umidade volumétrico residual
Função de conexão
Constante que descreve $\psi$ correspondente a $\theta_{\mathrm{r}}$
Coeficiente de permeabilidade saturado para um $\theta$ específico
Fator de igualdade
Coeficiente de permeabilidade saturado calculado
Tensão superficial da a água
Ângulo de contato
Viscosidade da água
Densidade da água
Constante de gravidade
Pendente da função de condutividade hidráulica não saturada
Volume da água
Volume total 
$\rho_{\mathrm{p}}$

e

$\alpha$

$\mathrm{R}_{\mathrm{i}}$

$\mathrm{r}_{\mathrm{i}}$

$\mathrm{n}_{\mathrm{i}}$

$\mathrm{S}_{\mathrm{C}}$

$\mathrm{S}_{\mathrm{a}}$

$\mathrm{S}_{\mathrm{r}}$

$\mathrm{h}_{\mathrm{co}}$

$\mathrm{D}_{10}$

$\mathrm{C}_{\mathrm{u}}$

$\mathrm{W}_{\mathrm{L}}$

V

i

$\mathrm{k}$

$\mathrm{H}$

$\mathrm{k}_{\mathrm{x}}, \mathrm{k}_{\mathrm{y}}$

Q

$\mathrm{t}$

FS

S

$\tau$

c, $\phi$

$c^{\prime}, \phi^{\prime}$

$\mathrm{k}_{\mathrm{h}}, \mathrm{k}_{\mathrm{v}}$
Densidade da partícula do solo

Ìndice de vazios

Constante da forma da partícula

Radio de uma partícula esférica equivalente à massa sólida $\mathrm{W}_{\mathrm{i}}$

Radio de poros de cada segmento

Número de partículas

Força capilar

Função de conexão

Grau de saturação

Elevação de capilaridade média

Diâmetro da partícula correspondente ao 10\% que passa

Coeficiente de uniformidade

Limite liquida (\%)

Velocidade de Darcy

Gradiente de carga hidráulica

Coeficiente de permeabilidade

Carga total

Coeficiente de permeabilidade na direção x e y

Fluxo de contorno

Tempo

Fator de segurança

Resistência ao cisalhamento

Tensão cisalhante induzida sob o potencial superfície de ruptura

Parâmetros de resistência

Parâmetros de resistência em termos de tensões efetivas

Coeficiente sismico vertical e horizontal 
Peso da massa do solo

$\mathrm{W}_{\mathrm{i}} \quad$ Peso da fatia de solo $\mathrm{i}$

$\mathrm{k}_{\mathrm{h}} \mathrm{W} \quad$ Força de inércia

$\mathrm{N}$

Força normal à base da fatia

S

Força tangente à base da fatia

$\mathrm{A}_{1}, \mathrm{~A}_{2} \quad$ Forças hidrostáticas

b Largura da fatia

$\mathrm{T}_{1}, \mathrm{~T}_{2} \quad$ Forças cisalhantes verticais interfatias

$E_{1}, E_{2} \quad$ Componente horizontal das forças entre as fatias

FS Fator de segurança

D Força aplicada na superficie do talude

l Comprimento da base da fatia

$\sigma_{\mathrm{n}} \quad$ Tensão normal média na base da fatia de comprimento unitário

$K_{0} \quad$ Coeficiente en repouso

$\mu_{\mathrm{a}} \quad$ Pressão de poros de ar

$\mu_{\mathrm{w}} \quad$ Pressão de poros de água

$\mathrm{f}_{0} \quad$ Fator de correção

$\mu \quad$ Poro-pressão médio na base da fatia

$\phi^{\mathrm{b}} \quad$ Ângulo que define o aumento na resistência cisalhante

$\mathrm{S}_{\mathrm{m}} \quad$ Parcela mobilizada da resistência ao cisalhamento

MEF Método dos elementos finitos 